\title{
Concentration and segregation of particles and bubbles by turbulence
}

\author{
E. Calzavarini ${ }^{1}$, M. Cencini ${ }^{2}$, D. Lohse ${ }^{1}$ and F. Toschi ${ }^{3}$
}

1 Dept. of Applied Physics, University of Twente, 7500 AE Enschede, The Netherlands. e.calzavarini@tnw.utwente.nl, lohse@tnw.utwente.nl

2 INFM-CNR, SMC Dept. of Physics, University of Rome La Sapienza, Piazzale A. Moro 2, 00185 Roma, Italy and CNR-ISC, Via dei Taurini 19, 00185 Roma, Italy. massimo.cencini@roma1.infn. it

3 CNR-IAC, Viale del Policlinico 137, I-00161 Roma, Italy and INFN, Sezione di Ferrara, Via G. Saragat 1, I-44100 Ferrara, Italy. toschi@iac.cnr.it

\section{The Problem}

Understanding the spatial distribution of finite-size massive particles, such as heavy impurities, dust, droplets, neutrally buoyant particles or bubbles suspended in incompressible, turbulent flows is a relevant issue in industrial engineering and atmospheric physics. In a turbulent flow vortices act as centrifuges ejecting particles heavier than the fluid and entrapping lighter ones $[1,2]$. This phenomenon produces on one side clusterization (also dubbed preferential concentration) on the other segregation (de-mixing) of particle species differing in size and densities.

Stated in a rather simplified form, i.e., assuming spherical, not-deformable particles smaller than the smallest scale of turbulence and gravity negligible, the equation of motion for a particle is [3]:

$$
\ddot{\mathbf{x}}=\beta\left(\partial_{t} \mathbf{u}+(\mathbf{u} \cdot \partial) \mathbf{u}\right)-(\dot{\mathbf{x}}-\mathbf{u}) / \tau \text {. }
$$

Here $\mathbf{u}=\mathbf{u}(\mathbf{x}(t), t)$ is the fluid velocity field described by the incompressible Navier-Stokes (NS) equation, while the parameters $\beta$ and $\tau$ account for the physical properties of the particle. Specifically, $\beta$ is a dimensionless number connected to the ratio between the particle density $\left(\rho_{p}\right)$ and the fluid one $\left(\rho_{f}\right)$, defined as $\beta \equiv 3 \rho_{f} /\left(\rho_{f}+2 \rho_{p}\right) . \tau$ instead is the typical particle response-time, that is $\tau \equiv a^{2} /(3 \beta \nu)$, with, $a$, the particle radius and, $\nu$, the fluid kinematic viscosity. Equation (1) coupled to NS can be considered an accurate physical model as long as the particle suspension is dilute, namely it is almost collisionless and it does not exert feedback on the fluid, that is to say, it is passively advected by the flow. 

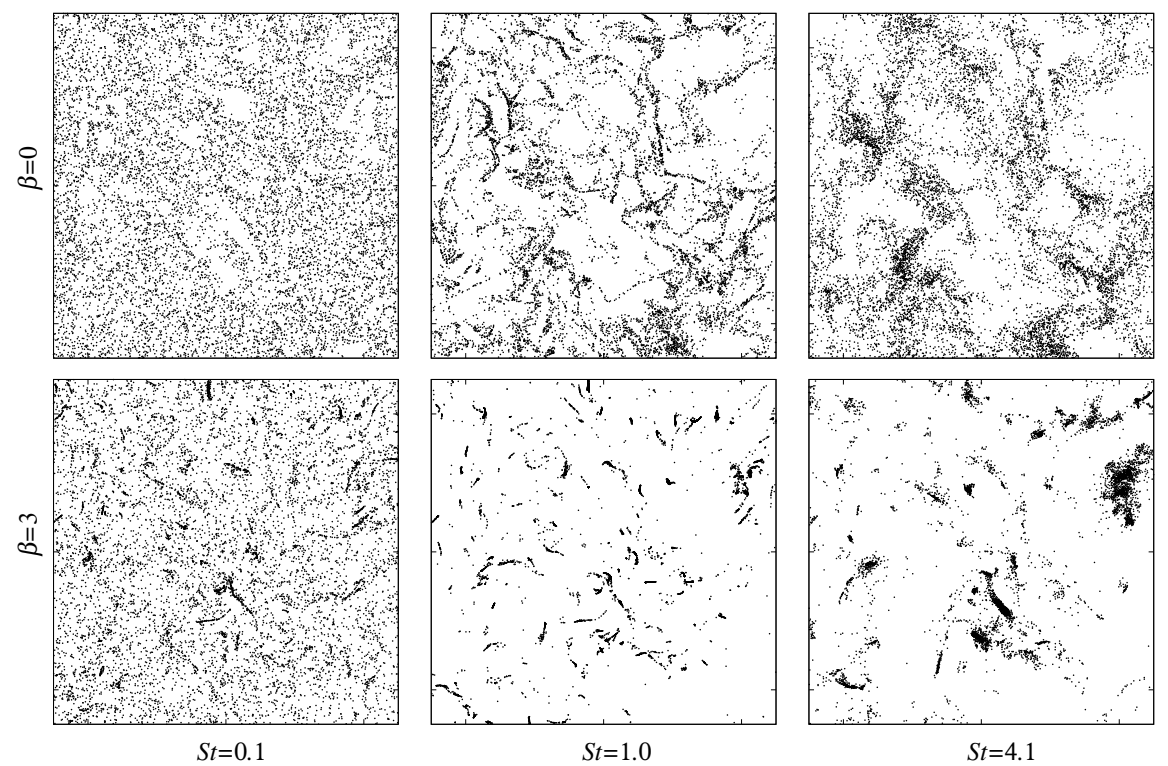

Fig. 1. Slices $320 \times 320 \times 8$ in size from a $512^{3}$ DNS. Both very heavy particles, $\beta=0$ (top), and bubbles, $\beta=3$ (bottom) at different Stokes numbers, $S t=0.1,1,4.1$ (left to right) are reported. The underlying fluid flow field is the same in all cases. All particles were injected homogeneously into the fluid domain roughly one large-eddyturnover-time before the snapshots.

\section{A numerical study}

We address the problem numerically. Here we present results from a series of direct numerical simulations (DNS) where passive suspensions of particles of variable density and size are tracked in a homogenous isotropic turbulent flow. We track up to $\sim 500$ sets of particles, corresponding to couples of values in the parameter-space $\beta$-St, where $S t \equiv \tau / \tau_{\eta}$ stands for the Stokes number and $\tau_{\eta}$ is the dissipative time-scale. The total number of particles per type ranges between $10^{5}-10^{6}$. Numerics are performed at different resolutions, $128^{3}$ and $512^{3}$, corresponding respectively to $R e_{\lambda}=65-185$, and extended in time for few large-eddy-turnover times. As shown in fig.1, non-homogeneities in the particle/bubble distributions, their dependence on the Stokes number and demixing between different species are already evident from plain visualizations.

Correlation dimension and concentration conditioned to flow topology

To gain better insight into the small-scale features of clustering, we study the probability, $P_{2}(r)$, that the distance between two particles is less than $r$. In the small-distance limit such probability has a power law behavior, $P_{2}(r) \sim r^{D_{2}}$. 
The exponent $D_{2}$, called correlation dimension of the spatial distribution, can be used as an estimator for the dimension of the set on which particles accumulates. Whether particle distribute locally uniformly $D_{2}$ equals the spatial dimension 3 . If instead $D_{2}<3$ we say that particles accumulate onto a fractal set.
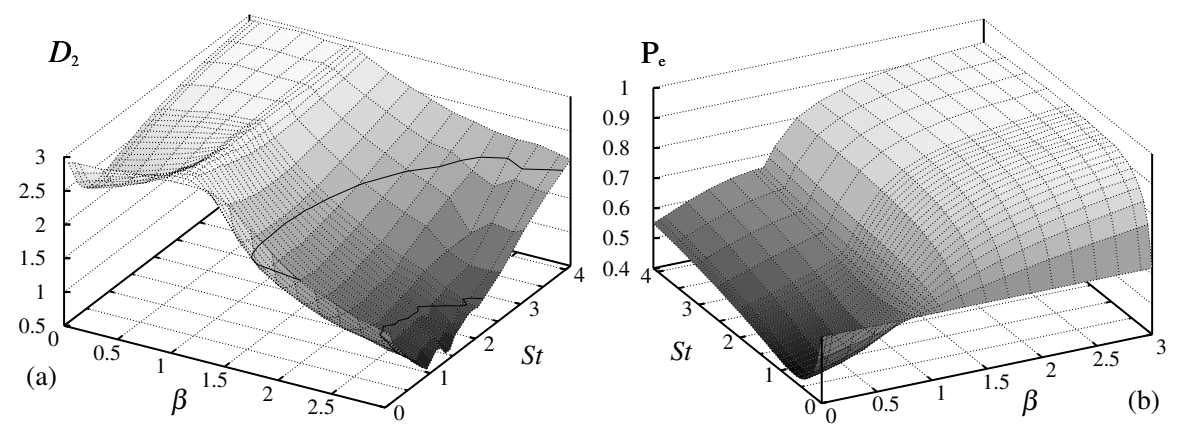

Fig. 2. (a) The correlation dimension $D_{2}$ as a function of the density parameter $\beta$ and the Stokes number. Isolines are drawn at $D_{2}=1,2$. (b) The probability $P_{e}$ to find a particle in elliptic regions of the flow versus $\beta$ and $S t$. Note that for fluid tracers $(S t=0$ particles $)$ it is here $P_{e} \simeq 0.6$, i.e., as expected elliptic regions in a turbulent flow extend over larger volumes than strain regions.

We observe that both heavy and light particles at small St numbers concentrate on fractal sets, see fig.2(a), the minimum of $D_{2}$ being around $S t \simeq 1$. Heavy particles always have $D_{2}$ above 2 , light particles instead reach even $D_{2}$ values below 1 . Indeed, the extremely strong agglomeration occurring for light particles produces here decimation of statistics and noisy $D_{2}$ results for $(\beta \gtrsim 2)$. Nevertheless, we may conclude that at small-scales filament-like clusters are expected for light particles while heavy particles agglomerate on surface-shaped regions.

Segregation is addressed by looking at particle concentrations conditioned to the local topology of the flow field. Vortical (also called elliptic) regions of the flow are defined as the positions where the eigenvalues of the local strain matrix $\left(\partial_{i} u_{j}\right)$ have imaginary parts [4]. The measure of the probability $\left(P_{e}\right)$ to find a particle, of given $\beta$-St value, in an elliptic region of the flow is reported in fig.2(b). Heavy particles are lacking in vortical regions, while extremely light particles concentrate almost completely in elliptic regions.

\section{References}

1. M. R. Maxey, J. Fluid Mech. 174, 441-465 (1987).

2. K. D. Squires, J. K. Eaton, Phys. Fluids A 3, 1169-1178 (1991).

3. M. R. Maxey, J. J. Riley, Phys. Fluids 26, 883-889 (1983).

4. J. Bec et al. J. Fluid Mech. 550, 349-358 (2006). 